Arab Univ. J. Agric. Sci., Ain Shams Univ., Cairo, 13(3), 755-769, 2005

\title{
EFFECT OF SALINITY AND NITROGEN BIO-FERTILIZATION ON SOME SUDAN GRASS (SORGHUM SUDANENSE (L.) MOENCH) VARIETIES AT RAS SUDR
}

[50]

\author{
Abd El-Rahman', S.M.; K.A. El Shouny²; M.A. Ashoub²; M.A. Abd El-Gawad ${ }^{1}$ \\ and M.Sh. Abd El-Maaboud ${ }^{1}$
}

\begin{abstract}
This study was carried out at Ras Sudr Experimental Station, South Sinai during 2001 and 2002 seasons. Four sudan grass varieties (Giza2, Piper, Hybrid102 and Is3214) were tested under five nitrogen fertilizer (Mineral and biofertilizer) treatments under two levels of irrigation water salinity (3700 and $9200 \mathrm{ppm}$ ). Growth characters i.e. plant height, number of tillers/plant, stem diameter, number of leaves/plant, leaf area, leaves/stem ratio, and forage yield (fresh and dry weight of stem + sheaths and fresh and dry weight of forage yield) were recorded. In addition, carbohydrates, protein, fibers and ash percentages (in leaves and stems) and proline in leaves were determined. Results demonstrate that the Piper variety had the highest value of forage yield compared with the other varieties. The recommended dose of mineral nitrogen fertilizer gave the highest values followed by mixture of biofertilizers (Azospirillum plus Azotobacter) under the two salinity levels of irrigation water at both cuts (the first one was harvested after 65 days from sowing date and the second was obtained at the same time interval). Moreover there was a significant decrease in all growth characteristics and the yield of four sudan grass varieties by increasing the level of irrigation water salinity from 3700 to $9200 \mathrm{ppm}$ except proline in leaves which significantly increased by increasing the level of salinity
\end{abstract}

Key words: Sorghum, (Sorghum sudanense), Salinity of irrigation water, Biofertilizers.

\section{INTRODUCTION}

Forage sudan grass (Sorghum sudanense (L.) Moench) is considered as one of the most important fodder crops in many countries of the world due to its high fodder yielding potential and good better quality. Sudan grass has excellent growing habit, quick growing regrowth after first cut and better palatability, digestibility, ratoonability and various forms of its utilization like green chop,

1- Desert Research Center, Matariya, Cairo, Egypt

2- Department of Agronomy, Faculty of Agriculture, Ain Shams University, Shoubra El-Kheima, Cairo, Egypt. 
Arab Univ. J. Agric. Sci., Ain Shams Univ., Cairo, 13(3), 755-769, 2005

silage and hay (Karwasra et al 1996 and

Dahiya et al 1997). In Egypt there is a great shortage in green forage in summer that considered being one of the main problems for feeding animals. Sudan grass is among the moderate crops to salinity tolerance therefore, it is important to develop new varieties, which are capable to grow under elevated salt levels in the soil and/or irrigation water (Francois et al 1984). Teosinte, Maize and Sudan grass are important forage crops which are grown extensively in salt affected semi-arid regions. Moreover sudan grass was comparatively more salt tolerant than Maize and Teosinte as far as various morpho-physiological characteristics are concerned (Kumar $\boldsymbol{e t}$ al 1991 and Datta et al 1996).

The soil and ground water salinity generally co-exist and have become a colossal agro-ecological problem associated with declining crop yield as reported by Hassan (1994) and Nassar et al (2000). Nitrogen fertilizers play an important role in increasing forage production of sudan grass with better nutritive value (Patel \& Rajagopal 2003 and Ramesh \& Sammi 2004). The cost of nitrogenous fertilizers is very high; hence, it becomes imperative to substitute nitrogen by some other cheaper sources, such as Azospirillum and/or Azotobacter which promote root growth and nitrogen fixation in soil, which may partially meet the nitrogen requirement of the crop (Patel et al 1992; Desale et al 1999 and Patidar \& Mali 2004). Subba Rao et al
(1979) demonstrated that application of Azospirillum and/or Azotobacter promoted root growth and more nitrogen fixation in soil, which help in increasing, fodder yield.

The main objective of the present investigation was to study the effect of nitrogen fertilizer (mineral and biofertilizer) treatments on yield and growth of sudan grass under high salinity conditions.

\section{MATERIAL AND METHODS}

This study was carried out at Ras Sudr Experimental Station, South Sinai during 2001 and 2002 seasons. Four sudan grass varieties namely; Giza2, Piper, Hybrid102 and Is3214 were chosen for the present study. Grains of the four varieties were provided by the Forage Research Division, Field Crops Research Institute of Agricultural Research Center, Ministry of Agriculture and Land Reclamation, Egypt. Efficient strains of Azotobacter chroococcum (AC), and Azospirillum brasilense (AB) were supplied by Microbiology unit, Desert Research Center, Egypt. The preceding winter crop was wheat. Sudan grass grains were sown on May $5^{\text {th }}$ at the two seasons. Experimental plot was four ridges, 3 meters long and $50 \mathrm{~cm}$ width. The size of each plot was $6 \mathrm{~m}^{2}$; the distance between hills was $15 \mathrm{~cm}$ on one side of the ridges. Split-split plot design

1- Desert Research Center, Matariya, Cairo, Egypt

2- Department of Agronomy, Faculty of Agriculture, Ain Shams University, Shoubra El-Kheima, Cairo, Egypt. 
with five replications was used. Irrigation water levels of salinity (3700 and 9200 ppm) occupied the main plots and nitrogen fertilizer the sub-plots, whereas, the four sudan grass varieties occupied the sub-sub plots. Mineral nitrogen fertilizer treatments were applied as:

a- Recommended rate; $60 \mathrm{~kg} \mathrm{~N} / \mathrm{fed}$. as ammonium nitrate $33.5 \% \mathrm{~N}$ added in two equal doses. The first one was applied after 20 and 50 days (half-and-half) and the second was applied after the first cut.

b- Without (control).

c- Azotobacter chrococcum (AC), rate of application five liters/fed. d- Azospirillum brasilense (AB), rate of application five liters/fed.

e- Mixture of (AC) and (AB), rate of application five liters/fed.

Two cuts were taken every season, the first one was harvested after 65 days from sowing date and the second was obtained at the same time interval. Phosphorus fertilizer as calcium super-phosphate $(15.5 \%)$ was added at a rate of 100 $\mathrm{kg} / \mathrm{fed}$. as a basal application during soil preparation and the other cultural practices were applied as recommended for growing sorghum in the area.

Mechanical and chemical properties of the soil are shown in Table (1) and chemical analysis of irrigation water at the two seasons is shown in Table (2).

Table 1. Mechanical and chemical properties of experimental soil at Ras Sudr in 2001 and 2002 growing seasons.

\begin{tabular}{|ccccc|}
\hline Season & \multicolumn{4}{c|}{ Physical analysis } \\
\cline { 2 - 4 } & \multicolumn{3}{c}{ Particle size distribution \% } & Class texture \\
\cline { 2 - 4 } & Sand & Silt & Clay & \\
\hline 2001 & 58.41 & 20.23 & 21.36 & Sandy loam \\
2002 & 62.34 & 17.15 & 20.51 & Sandy loam \\
\hline
\end{tabular}

\begin{tabular}{|ccccccccccccc|}
\hline \multicolumn{10}{c|}{ Chemical analysis } \\
\hline Season & \multicolumn{10}{c|}{ Cations $(\mathrm{mg} / \mathrm{L})$} \\
\cline { 2 - 11 } & $\mathrm{Ph}$ & $\begin{array}{c}\mathrm{Ec} \\
(\mathrm{ppm})\end{array}$ & $\mathrm{Ca}^{++}$ & $\mathrm{Mg}^{++}$ & $\mathrm{Na}^{+}$ & $\mathrm{K}^{+}$ & $\mathrm{CO}^{-}$ & $\mathrm{Hco3}^{-}$ & $\mathrm{Cl}^{-}$ & $\mathrm{SO}^{-}$ & $\mathrm{CaCO}^{-}$ \\
& & & & & & & & & & \\
\hline 2001 & 7.84 & 5510 & 19.01 & 47.31 & 18.32 & 0.67 & - & 6.51 & 51.03 & 27.47 & 49.37 \\
2002 & 7.72 & 5700 & 21.13 & 48.92 & 20.19 & 0.69 & - & 8.04 & 49.5 & 31.19 & 52.94 \\
\hline
\end{tabular}

Table 2. Chemical analysis of irrigation water at Ras Sudr in 2001 and 2002 growing seasons 
Arab Univ. J. Agric. Sci., Ain Shams Univ., Cairo, 13(3), 755-769, 2005

\begin{tabular}{|cccccccccccc|}
\hline Season & \multicolumn{9}{c|}{ Cations (mg/l) } & \multicolumn{5}{c|}{ Anions (mg/l) } \\
\cline { 2 - 10 } & $\mathrm{Ph}$ & $\begin{array}{c}\mathrm{Ec} \\
(\mathrm{ppm})\end{array}$ & $\mathrm{Ca}^{++}$ & $\mathrm{Mg}^{++}$ & $\mathrm{Na}^{+}$ & $\mathrm{K}^{+}$ & $\mathrm{CO}^{-}$ & $\mathrm{Hco3}^{-}$ & $\mathrm{Cl}^{-}$ & $\mathrm{SO}^{-}$ \\
\hline 2001 & 8.56 & 3700 & 40 & 75 & 33 & 0.28 & - & 8.0 & 65.51 & 74.01 \\
2002 & 8.35 & 9.200 & 35 & 70 & 37.4 & 0.31 & - & 9.5 & 62.02 & 71.21 \\
\hline
\end{tabular}

1- Desert Research Center, Matariya, Cairo, Egypt

2- Department of Agronomy, Faculty of Agriculture, Ain Shams University, Shoubra El-Kheima, Cairo, Egypt. 
Arab Univ. J. Agric. Sci., Ain Shams Univ., Cairo, 13(3), 755-769, 2005

Free proline in the leaves were determined according to the method described by Bates et al (1973). Total carbohydrate were determined in leaves and stems at first and second cuts according to the method described by Smith et al (1964). Total nitrogen was determined in leaves and stems as dry matter by using the modified micokieldahl method as described by Peach and Tracey (1956). Protein content was calculated by multiplying the total nitrogen by 6.25 . Crude fibers and ash contents were determined in leaves and stems according to the method described by (A.O.A.C. 1990). At cut, ten guarded plants were taken randomly from each plot of the five replicates to determine the growth characteristics at the two cutting stages. Combined analysis of the two growing seasons data was carried out according to procedure outlined by Steel and Torrie (1980). Duncan's multiple range test, (Duncan, 1955) was used to verify the significance of mean performance for all traits recorded.

\section{RESULTS AND DISCUSSION}

\section{Effect of irrigation water salinity and nitrogen fertilizers on some growth characters and forage yield}

Results given in Table (3) show that generally increasing salinity level of irrigation water from 3700 to 9200 ppm significantly decreased all of the growth characters of the four sudan grass varieties at the two cuts. Reduced growth levels under salinity conditions may be due to the water deficit as a result of water and osmotic potentials in the growth medium or to water and ionic disequilibrium in the aerial parts of the plant. Moreover, high concentration of salts may reduce the absorption capacity of roots. In this respect Kramer (1969) demonstrated that high concentration of salt cause a decrease in the permeability of roots to water, and hence a decrease in the rate of its entry into the plant. Kaoud and El-Fieshawy (1990) indicated that the $\mathrm{N}, \mathrm{P}, \mathrm{Ca}^{++}$and $\mathrm{K}^{+}$concentrations were decreased while $\mathrm{Na}^{+}$and $\mathrm{Mg}^{++}$ increased with increasing salt levels.

The interaction effect among salinity of irrigation water and nitrogen fertilizers on some growth characteristics of the four sudan grass varieties indicated that adding the recommended dose of mineral nitrogen fertilizer increased significantly plant height, fresh and dry weight of stem + sheaths/plant and leaves/stem ratio of Piper variety at the two cuts under $3700 \mathrm{ppm}$. On the other hand, the lowest mean values of plant height, was for Is 3214 followed by Hybrid102 under treatment without nitrogen fertilization when irrigated with saline water 9200ppm. Such trends were cited by Hassan (1994) and Karwasra and Dahiya (1997). Regarding, number of tillers per plant, Is3214 variety showed the highest mean values at the recommended dose of mineral nitrogen

1- Desert Research Center, Matariya, Cairo, Egypt

2- Department of Agronomy, Faculty of Agriculture, Ain Shams University, Shoubra El-Kheima, Cairo, Egypt. 
fertilizer followed by the mixture of biofertilizers with salinity of irrigation water 3700ppm at first and second cut. Meantime, there were no significant differences between the four sudan grass varieties at recommended dose of mineral nitrogen fertilizer with saline water $9200 \mathrm{ppm}$. These results could be attributed to the effect of high concentration of salts which caused an osmotic pressure that inhibited soluble nitrogen absorption and consequently prevent the stimulating effect of nitrogen on plant growth. Similar results were obtained by Ramamurthy (2002); Patel \& Rajagopal (2003) and Ramesh \& Sammi (2004). Concerning, stem diameter, results in Table (3) indicate that the highest mean values were obtained for Is3214 at the recommended dose of mineral nitrogen fertilizer followed by Azospirillum and mixture of biofertilizers at $3700 \mathrm{ppm}$ of irrigated water. On the other hand, Piper and Giza2 varieties had the higher stem diameter values at the recommended dose of mineral nitrogen fertilizer at salinity of irrigation water 9200ppm. Data presented in Table (3) show also that, Azospirillum brasilense (AB) strain was more effective and suitable strain for inoculation to the four sudan grass varieties as compared with Azotobacter chroococcum (AC) strain. Similar trends were obtained by Hassan (1994); Rawat \& Hazra (1998); Nassar et al (2000); Ramamurthy (2002) and Patidar and Mali (2004).

Regarding number of leaves/plant, (Table 3 ) results show that Piper variety had the highest values of number of leaves at the recommended dose of mineral nitrogen fertilizer and mixture of biofertilizers with salinity of irrigation water $3700 \mathrm{ppm}$ at the two cuts. While,
Hybrid102 variety recorded the highest values of number of leaves with applying the recommended nitrogen fertilizer at the first cut without significant differences. The lowest values were detected for Giza2 variety with salinity of irrigation water $3700 \mathrm{ppm}$ at the nitrogen control treatment at the two cuts. Piper variety took the same trend with adding recommended dose of mineral nitrogen fertilizer and mixture of biofertilizers under salinity of irrigation water 9200 ppm at the two cuts. Similar results were obtained by Hassan (1994); Karwasra \& Dahiya (1997) and Nassar et al (2000).

Concerning leaf area, data presented in Table (3) show that the lowest values were detected for Hybrid102 variety under treatment without nitrogen fertilization with water salinity $9200 \mathrm{ppm}$. On the other hand, the highest values were recorded by Is 3214 variety with the recommended dose of mineral nitrogen fertilizer followed by mixture of biofertilizers at salinity of irrigation water $3700 \mathrm{ppm}$ in the two cuts. These results agree those obtained by Saffa et al (1993) and Patidar \& Mali (2004). Hybrid 102 and Is3214 varieties had the lowest values for fresh and dry weight of stem+sheaths under treatment without nitrogen fertilization when irrigated with saline water $3700 \mathrm{ppm}$ in the two cuts (Table 4). Meantime, the highest values were recorded by Piper variety with salinity of irrigation water $3700 \mathrm{ppm}$ at the recommended dose of mineral nitrogen fertilizer treatment followed by mixture of biofertilizers in the two cuts. On the other hand, the highest values were recorded by Piper variety when irrigated with saline water 9200ppm with adding recommended dose of mineral 
nitrogen fertilizer followed by fertilizing with mixture of biofertilizers at the two cuts for fresh weight of stem + sheaths characters. Regarding dry weight of stem + sheaths, (Table 4) Piper variety fertilized with the recommended dose of mineral nitrogen fertilizer followed by mixture of biofertilizers had the highest values under salinity of irrigation water 9200 ppm at the two cuts. Similar results were found by Karwasra \& Dahiya (1997) and Nassar et al (2000).

Mean-time Piper variety had the highest values of leaves/stem ratio under 
Arab Univ. J. Agric. Sci., Ain Shams Univ., Cairo, 13(3), 755-769, 2005

Table 3. Effects of Salinity x N fertilizers x Varieties interaction on some growth characters of four Sudan grass varieties at Ras Sudr over two growing seasons (2001 and 2002).

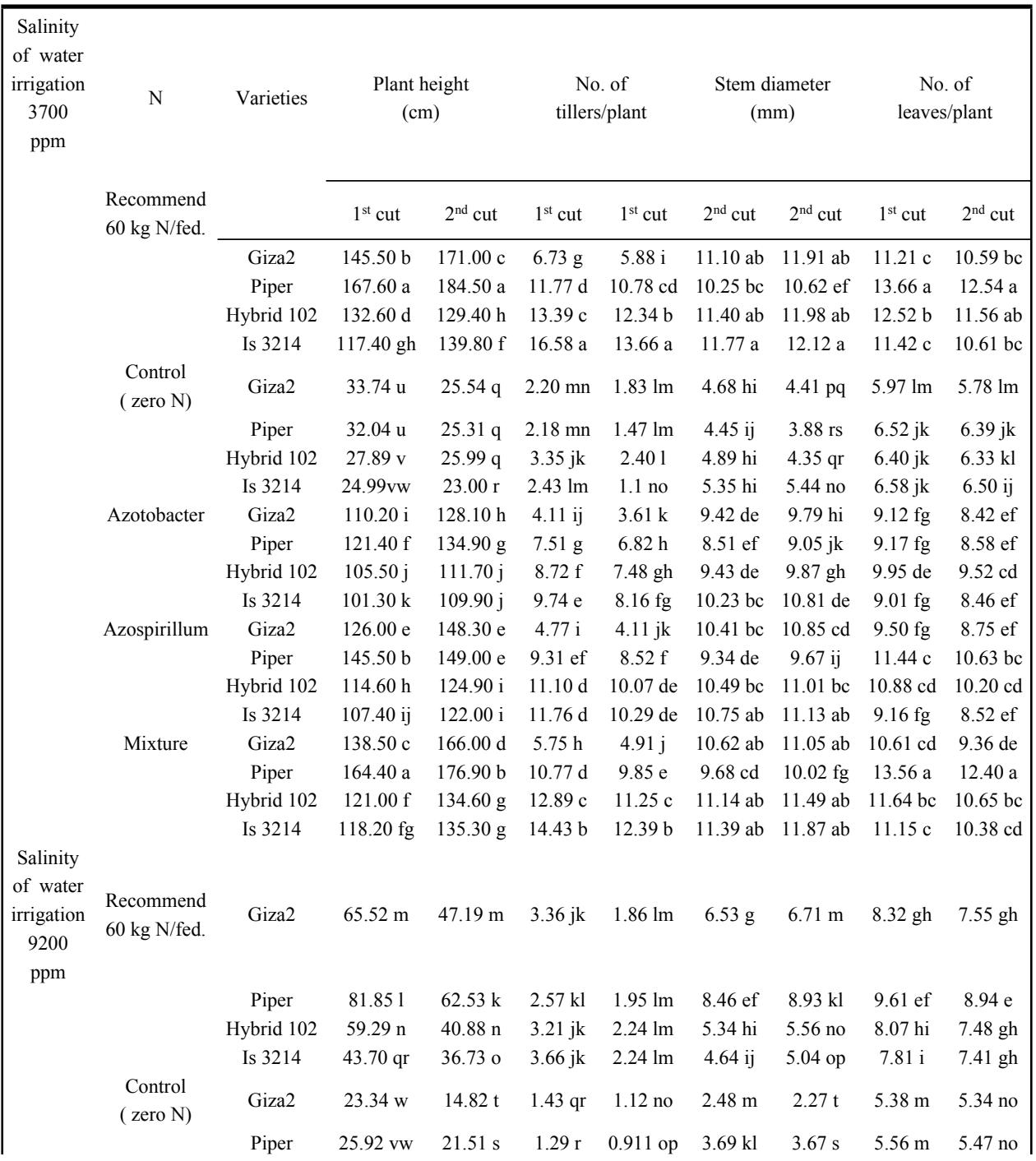

1- Desert Research Center, Matariya, Cairo, Egypt

2- Department of Agronomy, Faculty of Agriculture, Ain Shams University, Shoubra El-Kheima, Cairo, Egypt.

(Received May 25, 2005)

(Accepted June 6, 2005) 
Sudan grass yield at Ras Sudr

\begin{tabular}{|cccccccccc|} 
Azospirillum & Giza2 & $53.78 \mathrm{o}$ & $37.36 \mathrm{o}$ & $2.77 \mathrm{kl}$ & $1.90 \mathrm{~lm}$ & $5.34 \mathrm{hi}$ & $5.83 \mathrm{mn}$ & $7.24 \mathrm{ij}$ & $6.67 \mathrm{ij}$ \\
& Piper & $64.70 \mathrm{~m}$ & 50.431 & $2.06 \mathrm{no}$ & $1.40 \mathrm{mn}$ & $6.48 \mathrm{~g}$ & $6.40 \mathrm{mn}$ & $8.34 \mathrm{gh}$ & $7.65 \mathrm{fg}$ \\
& Hybrid 102 & $47.42 \mathrm{p}$ & $35.88 \mathrm{o}$ & $2.21 \mathrm{mn}$ & $1.57 \mathrm{~lm}$ & $3.82 \mathrm{jk}$ & $4.11 \mathrm{qr}$ & $7.53 \mathrm{ij}$ & $6.90 \mathrm{ij}$ \\
& Is 3214 & $37.29 \mathrm{t}$ & $29.82 \mathrm{p}$ & $1.84 \mathrm{no}$ & $1.19 \mathrm{no}$ & $3.64 \mathrm{kl}$ & $4.11 \mathrm{qr}$ & $6.32 \mathrm{kl}$ & $5.82 \mathrm{~lm}$ \\
Mixture & Giza2 & $62.52 \mathrm{mn}$ & $43.18 \mathrm{n}$ & $2.98 \mathrm{kl}$ & $2.26 \mathrm{~lm}$ & $5.83 \mathrm{gh}$ & $6.32 \mathrm{mn}$ & $8.13 \mathrm{hi}$ & $7.43 \mathrm{gh}$ \\
& Piper & 81.141 & $61.79 \mathrm{k}$ & $2.37 \mathrm{~lm}$ & $1.79 \mathrm{~lm}$ & $7.79 \mathrm{f}$ & 8.241 & $9.16 \mathrm{fg}$ & $8.40 \mathrm{ef}$ \\
& Hybrid 102 & $53.06 \mathrm{o}$ & $44.10 \mathrm{~m}$ & $2.65 \mathrm{kl}$ & $1.86 \mathrm{~lm}$ & $4.75 \mathrm{hi}$ & $5.09 \mathrm{op}$ & $8.18 \mathrm{hi}$ & $7.42 \mathrm{gh}$ \\
& Is 3214 & $41.17 \mathrm{rs}$ & $34.53 \mathrm{o}$ & $2.07 \mathrm{no}$ & $1.68 \mathrm{~lm}$ & $4.58 \mathrm{ij}$ & $4.94 \mathrm{op}$ & $7.24 \mathrm{ij}$ & $6.69 \mathrm{ij}$ \\
\hline
\end{tabular}

Arab Univ. J. Agric. Sci., 13(3), 2005 
Abdel-Rahman; El-Shouny; Ashoub; Abdel-Gawad and Abd El-Maaboud

Table 3. Cont.

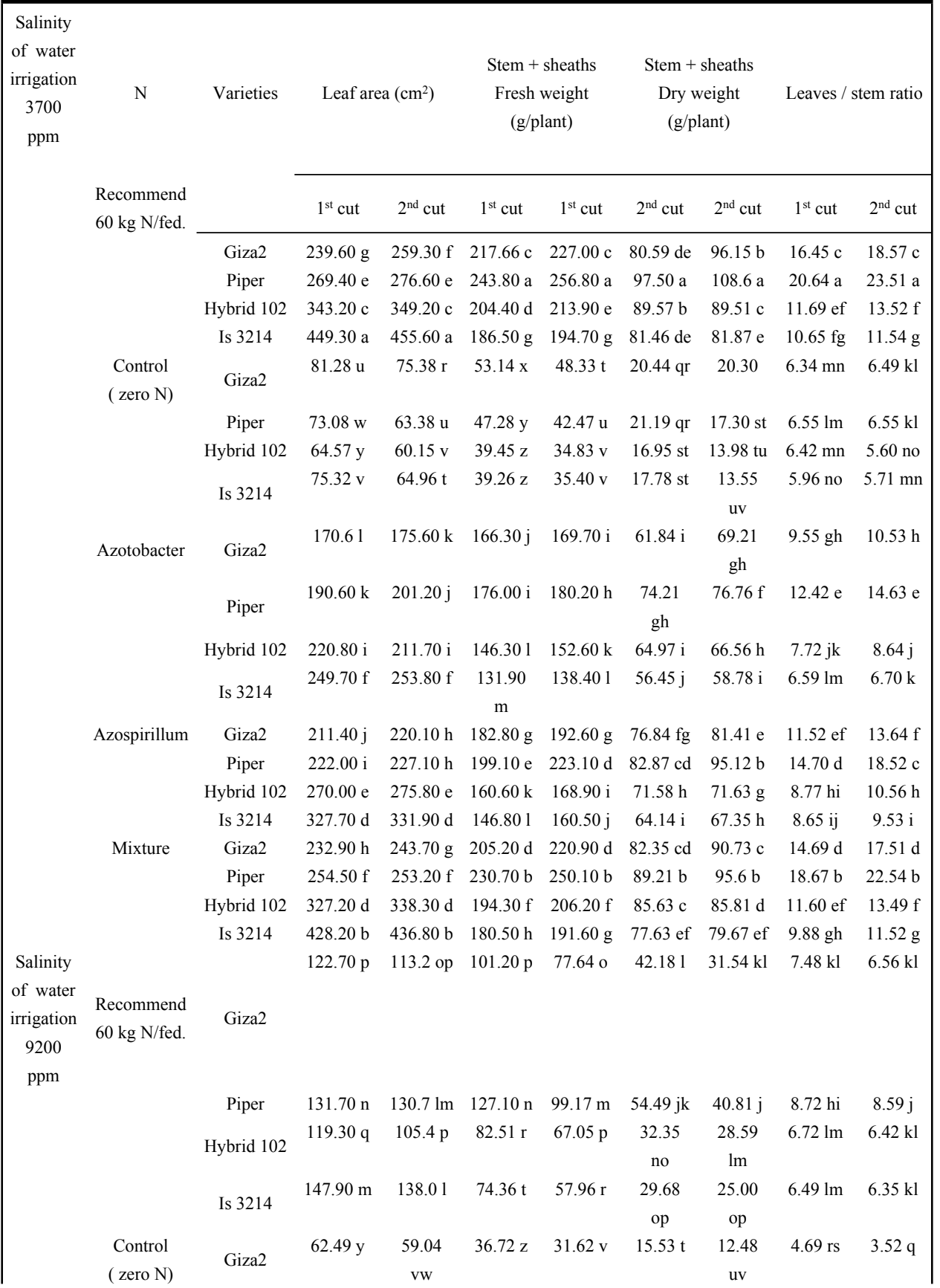

Arab Univ. J. Agric. Sci., 13(3), 2005 
11

Sudan grass yield at Ras Sudr

\begin{tabular}{|c|c|c|c|c|c|c|c|c|c|}
\hline & Piper & $72.25 \mathrm{x}$ & $\begin{array}{c}66.59 \\
\text { stu }\end{array}$ & $44.22 \mathrm{y}$ & $33.18 \mathrm{v}$ & $17.30 \mathrm{st}$ & $13.73 \mathrm{tu}$ & $4.55 \mathrm{rs}$ & $3.59 \mathrm{q}$ \\
\hline \multirow{4}{*}{ Azotobacter } & Hybrid 102 & $54.46 \mathrm{z}$ & $49.51 \mathrm{w}$ & $32.99 \mathrm{z}$ & $27.16 \mathrm{w}$ & $11.59 \mathrm{u}$ & $\begin{array}{c}11.24 \\
\text { uv }\end{array}$ & $4.42 \mathrm{st}$ & $3.08 \mathrm{qr}$ \\
\hline & Is 3214 & $57.98 \mathrm{z}$ & $52.31 \mathrm{w}$ & $30.89 \mathrm{z}$ & $24.39 \mathrm{w}$ & $11.80 \mathrm{u}$ & $10.63 \mathrm{v}$ & $3.93 \mathrm{t}$ & $2.63 \mathrm{r}$ \\
\hline & Giza2 & $79.18 \mathrm{v}$ & $71.84 \mathrm{rst}$ & $70.66 \mathrm{u}$ & $53.65 \mathrm{~s}$ & $\begin{array}{c}30.48 \\
\text { no }\end{array}$ & $20.91 \mathrm{qr}$ & $4.85 \mathrm{qr}$ & $4.54 \mathrm{p}$ \\
\hline & Piper & $86.03 \mathrm{u}$ & $80.36 \mathrm{r}$ & $91.15 \mathrm{q}$ & $63.08 \mathrm{q}$ & $\begin{array}{c}32.62 \\
\text { no }\end{array}$ & $\begin{array}{c}25.63 \\
\text { no }\end{array}$ & $5.51 \mathrm{op}$ & $4.63 \mathrm{p}$ \\
\hline \multirow{6}{*}{ Azospirillum } & Hybrid 102 & $74.85 \mathrm{w}$ & $\begin{array}{c}69.00 \\
\text { stu }\end{array}$ & $48.57 \mathrm{y}$ & $35.17 \mathrm{v}$ & $19.68 \mathrm{qr}$ & 14.84 tu & $4.63 \mathrm{rs}$ & $3.72 \mathrm{q}$ \\
\hline & Is 3214 & $101.30 \mathrm{t}$ & $92.19 \mathrm{q}$ & $44.49 \mathrm{y}$ & $35.33 \mathrm{v}$ & $18.55 \mathrm{rs}$ & $14.71 \mathrm{tu}$ & $4.50 \mathrm{st}$ & $3.59 \mathrm{q}$ \\
\hline & Giza2 & $100.40 \mathrm{t}$ & $90.84 \mathrm{q}$ & $79.66 \mathrm{r}$ & $67.32 p$ & $\begin{array}{c}34.42 \\
\mathrm{mn}\end{array}$ & $\begin{array}{c}27.70 \\
\mathrm{mn}\end{array}$ & 5.80 no & $5.56 \mathrm{o}$ \\
\hline & Piper & $111.00 \mathrm{~s}$ & $105.7 \mathrm{p}$ & $103.10 \mathrm{p}$ & $78.09 \mathrm{o}$ & 43.421 & $34.11 \mathrm{k}$ & $6.54 \mathrm{~lm}$ & $6.18 \mathrm{kl}$ \\
\hline & Hybrid 102 & $85.53 \mathrm{u}$ & $77.46 \mathrm{rs}$ & $61.03 \mathrm{w}$ & $46.75 \mathrm{t}$ & $22.56 \mathrm{qr}$ & $19.91 \mathrm{rs}$ & $5.35 \mathrm{pq}$ & $4.70 \mathrm{p}$ \\
\hline & Is 3214 & $116.70 \mathrm{r}$ & $106.3 \mathrm{p}$ & $55.21 \mathrm{x}$ & $45.54 \mathrm{t}$ & $23.41 \mathrm{q}$ & $19.38 \mathrm{rs}$ & $5.52 \mathrm{op}$ & $4.54 \mathrm{p}$ \\
\hline \multirow[t]{4}{*}{ Mixture } & Giza2 & $113.90 \mathrm{r}$ & $108.2 \mathrm{p}$ & $94.10 \mathrm{q}$ & 74.30 o & $36.68 \mathrm{~m}$ & $30.80 \mathrm{kl}$ & $6.99 \mathrm{~lm}$ & $6.60 \mathrm{k}$ \\
\hline & Piper & $127.70 \mathrm{o}$ & 119.8 no & $121.90 \mathrm{o}$ & $92.03 \mathrm{n}$ & $52.14 \mathrm{k}$ & $38.22 \mathrm{j}$ & $8.53 \mathrm{ij}$ & $8.13 \mathrm{j}$ \\
\hline & Hybrid 102 & $103.50 \mathrm{t}$ & $94.16 \mathrm{q}$ & $76.68 \mathrm{~s}$ & $62.84 \mathrm{q}$ & $27.73 \mathrm{p}$ & $\begin{array}{c}22.22 \\
\mathrm{pq}\end{array}$ & $6.64 \mathrm{~lm}$ & $5.74 \mathrm{~lm}$ \\
\hline & Is 3214 & $135.50 \mathrm{n}$ & $\begin{array}{c}127.3 \\
\mathrm{mn}\end{array}$ & $68.43 \mathrm{v}$ & $55.34 \mathrm{r}$ & $\begin{array}{c}29.38 \\
\text { op }\end{array}$ & $\begin{array}{c}24.22 \\
\text { op }\end{array}$ & $6.35 \mathrm{mn}$ & 5.61 no \\
\hline
\end{tabular}

Arab Univ. J. Agric. Sci., 13(3), 2005 
12

Abdel-Rahman; El-Shouny; Ashoub; Abdel-Gawad and Abd El-Maaboud

Table 4. Effects of Salinity x N fertilizers x Varieties interaction on yield, carbohydrate percentage of four sudan grass varieties at Ras Sudr over 2001 and 2002 growing seasons.

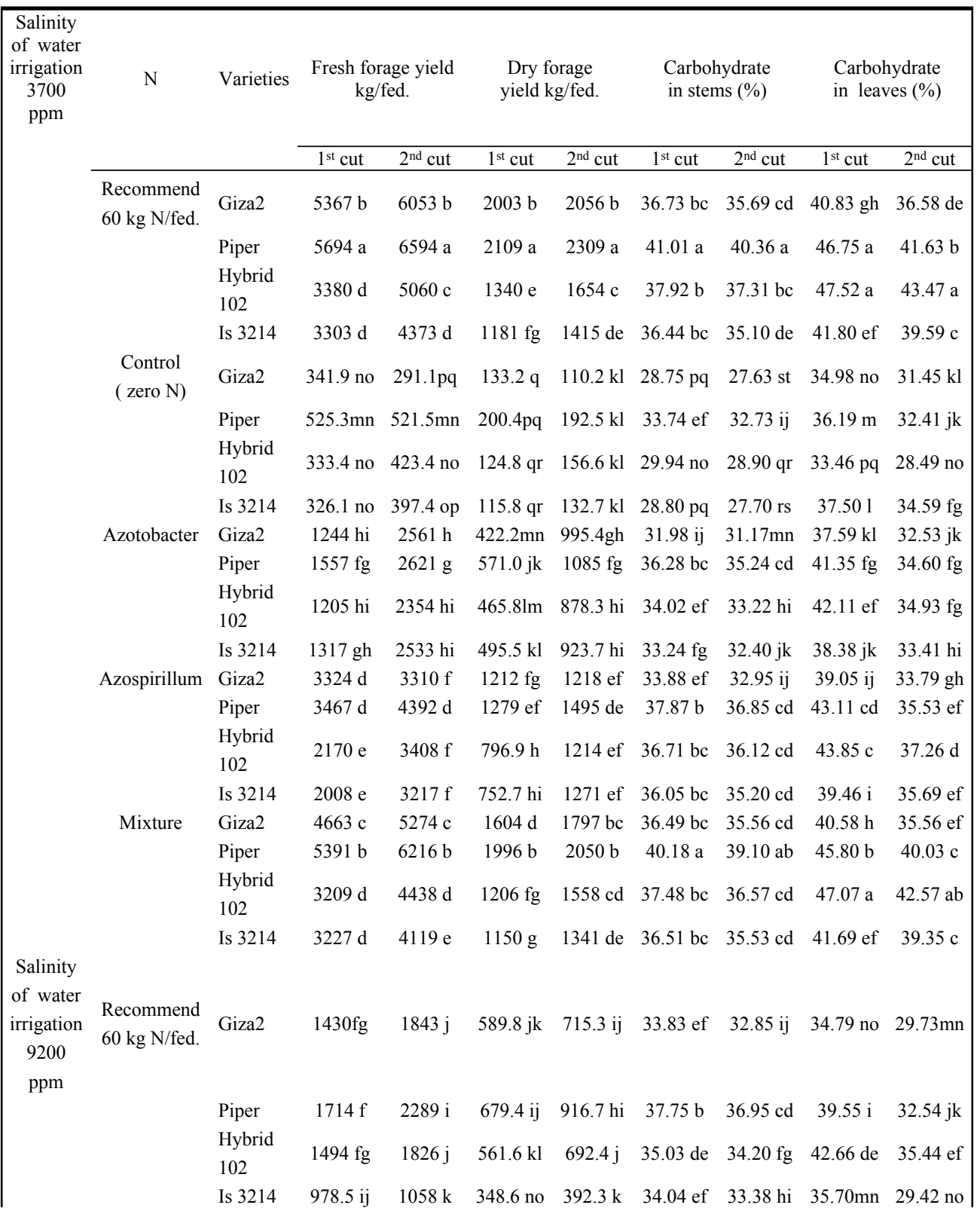

Arab Univ. J. Agric. Sci., 13(3), 2005 
13

\section{Sudan grass yield at Ras Sudr}

\begin{tabular}{|c|c|c|c|c|c|c|c|c|c|}
\hline Control & iza2 & 104.3 o & $94.50 \mathrm{q}$ & $40.53 \mathrm{~s}$ & 38.11 & $26.88 \mathrm{rs}$ & $25.71 \mathrm{uv}$ & $30.71 \mathrm{u}$ & $24.72 \mathrm{rs}$ \\
\hline \multirow{7}{*}{ Azotobacter } & per & $124.8 \mathrm{o}$ & $109.9 \mathrm{q}$ & $55.79 \mathrm{rs}$ & 40.81 & $27.83 \mathrm{qr}$ & $26.93 \mathrm{tu}$ & $29.65 \mathrm{v}$ & $24.44 \mathrm{rs}$ \\
\hline & $\begin{array}{l}\text { Hybrid } \\
102\end{array}$ & 99.13 о & $93.25 \mathrm{q}$ & $40.35 \mathrm{~s}$ & 38.421 & $27.03 \mathrm{qr}$ & $25.90 \mathrm{uv}$ & $31.62 \mathrm{st}$ & $23.69 \mathrm{~s}$ \\
\hline & Is 3214 & $96.99 \mathrm{o}$ & $97.77 \mathrm{q}$ & $42.46 \mathrm{~s}$ & 39.51 & $25.85 \mathrm{~s}$ & $24.90 \mathrm{v}$ & 30.99 tu & $24.42 \mathrm{rs}$ \\
\hline & Giza2 & $497.6 \mathrm{mn}$ & $603.8 \mathrm{mn}$ & 173.5 po & $235.8 \mathrm{kl}$ & $30.74 \mathrm{~lm}$ & 29.70 op & $31.48 \mathrm{tu}$ & $25.12 \mathrm{r}$ \\
\hline & Piper & $632.31 \mathrm{~m}$ & $693.51 \mathrm{~m}$ & 234.0 op & $260.0 \mathrm{kl}$ & $31.96 \mathrm{ij}$ & $31.24 \mathrm{mn}$ & $32.51 \mathrm{qr}$ & $26.45 \mathrm{p}$ \\
\hline & $\begin{array}{l}\text { Hybrid } \\
102\end{array}$ & 405.0 no & $478.6 \mathrm{mn}$ & $144.4 \mathrm{qr}$ & $165.5 \mathrm{kl}$ & $30.09 \mathrm{mn}$ & $29.24 \mathrm{pq}$ & $36.47 \mathrm{~m}$ & 28.73 no \\
\hline & Is 3214 & 334.1 no & 345.9 op & $117.9 \mathrm{qr}$ & $148.1 \mathrm{kl}$ & 29.82 op & $28.87 \mathrm{qr}$ & $31.90 \mathrm{rs}$ & $25.24 \mathrm{qr}$ \\
\hline \multirow[t]{4}{*}{ Azospirillum } & Giza2 & $595.81 \mathrm{~m}$ & $708.31 \mathrm{~m}$ & 233.2 op & $284.8 \mathrm{kl}$ & $31.18 \mathrm{kl}$ & 30.07 no & $32.61 \mathrm{qr}$ & $26.48 \mathrm{p}$ \\
\hline & Piper & $759.0 \mathrm{kl}$ & $869.0 \mathrm{kl}$ & 280.6 op & $348.4 \mathrm{kl}$ & $35.67 \mathrm{~cd}$ & $34.65 \mathrm{ef}$ & $33.76 \mathrm{p}$ & 28.76 no \\
\hline & $\begin{array}{l}\text { Hybrid } \\
102\end{array}$ & $505.5 \mathrm{mn}$ & $582.8 \mathrm{mn}$ & $192.2 \mathrm{pq}$ & $222.2 \mathrm{kl}$ & $31.85 \mathrm{jk}$ & $31.15 \mathrm{mn}$ & $38.57 \mathrm{ij}$ & $30.83 \mathrm{~lm}$ \\
\hline & Is 3214 & $432.4 \mathrm{n}$ & $489.9 \mathrm{mn}$ & $167.1 \mathrm{pq}$ & $177.7 \mathrm{kl}$ & $31.17 \mathrm{kl}$ & 30.29 no & $33.41 \mathrm{pq}$ & $26.33 \mathrm{pq}$ \\
\hline \multirow[t]{4}{*}{ Mixture } & Giza2 & $880.3 \mathrm{kl}$ & $1071 \mathrm{k}$ & 321.7 no & $405.7 \mathrm{k}$ & $32.75 \mathrm{gh}$ & $31.69 \mathrm{~lm}$ & 34.14 op & $28.36 \mathrm{o}$ \\
\hline & Piper & $1430 \mathrm{fg}$ & $1950 \mathrm{j}$ & $575.5 \mathrm{jk}$ & $730.1 \mathrm{ij}$ & $37.10 \mathrm{bc}$ & $36.11 \mathrm{~cd}$ & $37.75 \mathrm{kl}$ & $32.65 \mathrm{ij}$ \\
\hline & $\begin{array}{l}\text { Hybrid } \\
102\end{array}$ & $945.6 \mathrm{jk}$ & $923.5 \mathrm{kl}$ & 349.6 no & $333.8 \mathrm{kl}$ & $34.73 \mathrm{de}$ & $33.96 \mathrm{gh}$ & 41.84 ef & 35.72 ef \\
\hline & Is 3214 & $748.3 \mathrm{kl}$ & $922.0 \mathrm{kl}$ & 286.2 op & $348.0 \mathrm{kl}$ & $32.63 \mathrm{hi}$ & $32.00 \mathrm{kl}$ & $35.68 \mathrm{mn}$ & $29.64 \mathrm{mn}$ \\
\hline
\end{tabular}

Arab Univ. J. Agric. Sci., 13(3), 2005 
Arab Univ. J. Agric. Sci., Ain Shams Univ., Cairo, 13(3), 755-769, 2005

salinity of irrigation water of $3700 \mathrm{ppm}$ with the recommended dose of mineral nitrogen fertilizer followed by mixture of biofertilizers at the two cuts (Table 3). Whereas, the lowest values were recorded by Is 3214 variety with using salinity of irrigation water $9200 \mathrm{ppm}$ under treatment without nitrogen fertilization. Similar results were obtained by Hassan (1994) and Nassar et al (2000). Data in Table (4) demonstrated that the high values of fresh and dry weight of forage yield/fed., were recorded by Piper variety under salinity of irrigation water of $3700 \mathrm{ppm}$ at the recommended dose of mineral nitrogen fertilizer, followed by mixture of biofertilizers of the same variety at the two cuts. Meantime, Piper variety had the maximum mean values of fresh and dry weight of forage yield/fed., at the recommended dose of mineral nitrogen fertilizer followed by mixture of biofertilizers with adding saline water $9200 \mathrm{ppm}$ of the same variety at the two cuts. The minimum values of fresh and dry weight of forage yield/fed., were obtained by Is 3214 , Hybrid102, Giza2 and Piper varieties under treatment without nitrogen fertilization at salinity of irrigation water $9200 \mathrm{ppm}$ in both cuts. Such results confirm those of Patel et al (1992); Barik et al (1998); Panwar et al (1999) and Kaoud \& El-Fieshawy (1990), who indicated that sorghum is a moderately salt tolerant plant. The N, P, $\mathrm{Ca}^{++}$and $\mathrm{K}^{+}$concentrations were decreased while $\mathrm{Na}^{+}$and $\mathrm{Mg}^{++}$increased with increasing salt levels.

\section{Effect of irrigation water salinity and nitrogen fertilizers on some chemical components}

The average values of total carbohydrates, protein, proline, fibers and ash percentages are shown in Tables (4 and 5). Data show that, high level of salinity (9200ppm) decreased the mean values of all these chemical contents except of proline percentage in leaves at first cut whereas, insignificant increase in such chemical components has been recorded by decreasing the level of salinity from (9200 to $3700 \mathrm{ppm}$ ). On the other hand, proline percentage in leaves increased by increasing the salinity of irrigation water from (3700 to $9200 \mathrm{ppm}$ ), similar results were mentioned by

Hassan (1994) and Nassar et al (2000) who indicated that proline helps in osmoregulation and protects the cells against salinity stress. The highest mean values of total carbohydrates percentages in stem was found in Piper variety at the recommended dose of mineral nitrogen fertilizer followed by treatment mixture of biofertilizers on salinity of irrigation water (3700ppm). Hybrid102 had the highest mean values of total carbohydrates percentages in leaves with adding the recommended dose of mineral nitrogen fertilizer and mixture of biofertilizers at the two cuts and Piper variety at the recommended dose of mineral nitrogen fertilizer in the first cut. However Is3214 variety had the lowest significant values of total carbohydrates percentages in stems and leaves under

1- Desert Research Center, Matariya, Cairo, Egypt

2- Department of Agronomy, Faculty of Agriculture, Ain Shams University, Shoubra El-Kheima, Cairo, Egypt. 
15

Sudan grass yield at Ras Sudr

treatment without nitrogen fertilizer on salinity of irrigation water (9200ppm) in the both cuts, as shown in Table (4).

These results are in harmony with those obtained by Mustafa \& Shaheen (1984); Abbas et al (1993); Hassan (1994);
Nassar et al (2000) and Ram \& Bhagwan (2003).

Data in Table (5) show that the protein and fiber percentages in leaves and stems of the four sudan grass varieties significantly differed in there response to 
Arab Univ. J. Agric. Sci., Ain Shams Univ., Cairo, 13(3), 755-769, 2005

Table 5. Effects of Salinity x N fertilizers x Varieties interaction on some chemical properties of four Sudan grass varieties at Ras Sudr over 2001 and 2002 growing seasons.

\begin{tabular}{|c|c|c|c|c|c|c|c|c|c|}
\hline \multirow{2}{*}{$\begin{array}{c}\text { Salinity } \\
\text { of water } \\
\text { irrigation } \\
3700 \\
\text { ppm }\end{array}$} & \multirow[t]{2}{*}{$\mathrm{N}$} & \multirow[t]{2}{*}{ Varieties } & \multicolumn{3}{|c|}{$\begin{array}{c}\text { Protein } \\
\text { in stems }(\%)\end{array}$} & \multicolumn{2}{|c|}{$\begin{array}{c}\text { Fiber } \\
\text { in leaves }(\%)\end{array}$} & \multicolumn{2}{|c|}{$\begin{array}{c}\text { Proline } \\
\text { in leaves }(\mu \mathrm{m} / \mathrm{g})\end{array}$} \\
\hline & & & $1^{\text {st }}$ cut & $2^{\text {nd }}$ cut & $1^{\text {st }}$ cut & $2^{\text {nd }}$ cut & $1^{\text {st }}$ cut & $2^{\text {nd }}$ cut & $1^{\text {st }}$ cut \\
\hline & $\begin{array}{l}\text { Recommend } \\
60 \mathrm{~kg} \mathrm{~N} / \text { fed. }\end{array}$ & Giza2 & $12.06 \mathrm{ab}$ & $12.41 \mathrm{ab}$ & $\begin{array}{c}6.85 \\
\mathrm{ab}\end{array}$ & $\begin{array}{c}7.09 \\
\mathrm{ab}\end{array}$ & $\begin{array}{c}23.55 \\
b c\end{array}$ & $\begin{array}{c}22.72 \\
\text { bc }\end{array}$ & $1.873 \mathrm{hi}$ \\
\hline & & Piper & $12.56 \mathrm{a}$ & $12.92 \mathrm{a}$ & $7.49 \mathrm{a}$ & $7.59 \mathrm{a}$ & $26.10 \mathrm{a}$ & $25.72 \mathrm{a}$ & $\begin{array}{c}2.259 \\
\mathrm{de}\end{array}$ \\
\hline & & Hybrid 102 & $11.30 \mathrm{bc}$ & $11.60 \mathrm{bc}$ & $\begin{array}{c}6.38 \\
b c\end{array}$ & $\begin{array}{c}6.52 \\
\text { bc }\end{array}$ & $\begin{array}{c}22.42 \\
\mathrm{~cd}\end{array}$ & $\begin{array}{c}21.99 \\
\mathrm{~cd}\end{array}$ & $\begin{array}{c}1.126 \\
\mathrm{mn}\end{array}$ \\
\hline & & Is 3214 & $10.21 \mathrm{~d}$ & $10.51 \mathrm{~d}$ & $\begin{array}{c}6.44 \\
b c\end{array}$ & $\begin{array}{c}6.59 \\
\text { bc }\end{array}$ & $\begin{array}{c}20.67 \\
\text { de }\end{array}$ & $\begin{array}{c}20.34 \\
\text { de }\end{array}$ & $\begin{array}{c}1.224 \\
1 \mathrm{~m}\end{array}$ \\
\hline & $\begin{array}{c}\text { Control } \\
(\text { zero } \mathrm{N})\end{array}$ & Giza2 & $9.10 \mathrm{ef}$ & 9.44 ef & $3.51 \mathrm{kl}$ & $\begin{array}{c}3.64 \\
\text { op }\end{array}$ & $\begin{array}{c}16.80 \\
\mathrm{mn}\end{array}$ & $\begin{array}{c}16.72 \\
\operatorname{lm}\end{array}$ & $0.428 \mathrm{r}$ \\
\hline & & Piper & $8.20 \mathrm{gh}$ & $8.52 \mathrm{gh}$ & $3.77 \mathrm{kl}$ & $\begin{array}{c}3.91 \\
\text { no }\end{array}$ & $18.33 \mathrm{jk}$ & $17.99 \mathrm{jk}$ & $0.502 \mathrm{r}$ \\
\hline & & Hybrid 102 & $7.30 \mathrm{ij}$ & $7.58 \mathrm{hi}$ & $\begin{array}{c}3.01 \\
\text { op }\end{array}$ & $3.10 \mathrm{rs}$ & $\begin{array}{c}16.97 \\
\mathrm{mn}\end{array}$ & $\begin{array}{c}16.61 \\
\mathrm{mn}\end{array}$ & $0.346 \mathrm{r}$ \\
\hline & & Is 3214 & $6.27 \mathrm{mn}$ & $6.50 \mathrm{~lm}$ & $\begin{array}{c}3.42 \\
\mathrm{mn}\end{array}$ & $\begin{array}{c}3.48 \\
\mathrm{pq}\end{array}$ & $\begin{array}{c}15.11 \\
\text { op }\end{array}$ & $\begin{array}{c}14.64 \\
\text { op }\end{array}$ & $0.350 \mathrm{r}$ \\
\hline & Azotobacter & Giza2 & $9.22 \mathrm{e}$ & $9.72 \mathrm{de}$ & $\begin{array}{l}5.20 \\
\mathrm{fg}\end{array}$ & $\begin{array}{c}5.36 \\
\mathrm{gh}\end{array}$ & $18.93 \mathrm{hi}$ & $18.49 \mathrm{ij}$ & $0.793 \mathrm{p}$ \\
\hline & & Piper & $9.26 \mathrm{e}$ & $9.51 \mathrm{ef}$ & 5.46 ef & $\begin{array}{c}5.56 \\
\text { fg }\end{array}$ & $\begin{array}{c}20.30 \\
\mathrm{fg}\end{array}$ & $19.96 \mathrm{ef}$ & $\begin{array}{c}1.118 \\
\mathrm{mn}\end{array}$ \\
\hline & & Hybrid 102 & $8.30 \mathrm{fg}$ & $8.54 \mathrm{gh}$ & $4.53 \mathrm{hi}$ & $4.72 \mathrm{jk}$ & $\begin{array}{c}19.39 \\
\mathrm{fg}\end{array}$ & $\begin{array}{c}19.04 \\
\text { gh }\end{array}$ & $\begin{array}{c}0.559 \\
\mathrm{qr}\end{array}$ \\
\hline & & Is 3214 & $7.34 \mathrm{hi}$ & $7.62 \mathrm{hi}$ & $4.46 \mathrm{ij}$ & $4.59 \mathrm{kl}$ & $17.91 \mathrm{kl}$ & $17.55 \mathrm{kl}$ & $\begin{array}{c}0.763 \\
\mathrm{pq}\end{array}$ \\
\hline & Azospirillum & Giza2 & $10.21 \mathrm{~d}$ & $10.55 \mathrm{~d}$ & $\begin{array}{c}6.15 \\
\mathrm{~cd}\end{array}$ & $\begin{array}{c}6.31 \\
b c\end{array}$ & $\begin{array}{c}20.08 \\
\mathrm{fg}\end{array}$ & $\begin{array}{c}19.67 \\
\mathrm{fg}\end{array}$ & $1.138 \mathrm{~m}$ \\
\hline & & Piper & $10.24 \mathrm{~d}$ & $10.53 \mathrm{~d}$ & $\begin{array}{c}5.87 \\
\mathrm{~cd}\end{array}$ & $\begin{array}{c}6.01 \\
\text { de }\end{array}$ & $\begin{array}{c}21.08 \\
\text { de }\end{array}$ & $\begin{array}{c}20.75 \\
\text { de }\end{array}$ & $1.385 \mathrm{kl}$ \\
\hline & & Hybrid 102 & $9.29 \mathrm{e}$ & 9.49 ef & $\begin{array}{c}5.91 \\
\mathrm{~cd} \\
5.61\end{array}$ & $\begin{array}{c}6.02 \\
\text { de }\end{array}$ & $\begin{array}{c}20.93 \\
\text { de } \\
19.77\end{array}$ & $\begin{array}{c}20.60 \\
\text { de } \\
19.46\end{array}$ & $\begin{array}{c}0.878 \\
\text { no } \\
0.855\end{array}$ \\
\hline
\end{tabular}

1- Desert Research Center, Matariya, Cairo, Egypt

2- Department of Agronomy, Faculty of Agriculture, Ain Shams University, Shoubra El-Kheima, Cairo, Egypt. 


\section{Sudan grass yield at Ras Sudr}

Salinity

of water

irrigation

9200

Recommend

$60 \mathrm{~kg} \mathrm{~N} /$ fed.

Giza2

$9.29 \mathrm{e} \quad 9.35 \mathrm{fg}$

$\begin{array}{ccccc}6.78 & 6.87 & 23.02 & 22.66 & 1.083 \\ \mathrm{ab} & \mathrm{ab} & \mathrm{bc} & \mathrm{bc} & \mathrm{mn} \\ 6.51 & 6.69 & 20.62 & 20.28 & 0.996 \\ \mathrm{bc} & \mathrm{ab} & \mathrm{de} & \mathrm{de} & \mathrm{mn}\end{array}$

ppm

\begin{tabular}{|c|c|c|c|c|c|c|c|c|}
\hline & Piper & $10.14 \mathrm{~d}$ & $10.33 \mathrm{de}$ & $\begin{array}{c}6.88 \\
a b\end{array}$ & $\begin{array}{c}6.99 \\
\mathrm{ab}\end{array}$ & $\begin{array}{c}22.18 \\
\mathrm{~cd}\end{array}$ & $\begin{array}{c}21.74 \\
\mathrm{~cd}\end{array}$ & $3.371 \mathrm{a}$ \\
\hline & Hybrid 102 & $8.17 \mathrm{gh}$ & $8.35 \mathrm{hi}$ & $\begin{array}{l}5.18 \\
\text { fg }\end{array}$ & $5.24 \mathrm{hi}$ & $\begin{array}{c}20.92 \\
\text { de }\end{array}$ & $\begin{array}{c}20.61 \\
\text { de }\end{array}$ & $2.619 \mathrm{c}$ \\
\hline \multirow{5}{*}{$\begin{array}{c}\text { Control } \\
\text { ( zero } \mathrm{N})\end{array}$} & Is 3214 & 8.14 hi & $8.39 \mathrm{hi}$ & $4.46 \mathrm{ij}$ & $\begin{array}{c}4.51 \\
\operatorname{lm}\end{array}$ & $\begin{array}{c}20.24 \\
\mathrm{fg}\end{array}$ & 19.87 ef & $\begin{array}{c}2.229 \\
\text { de }\end{array}$ \\
\hline & Giza2 & $6.22 \mathrm{n}$ & $6.38 \mathrm{n}$ & $\begin{array}{c}2.94 \\
\text { op }\end{array}$ & $3.00 \mathrm{st}$ & $\begin{array}{c}15.05 \\
\text { op }\end{array}$ & $\begin{array}{c}14.56 \\
\text { op }\end{array}$ & $1.400 \mathrm{kl}$ \\
\hline & Piper & $7.07 \mathrm{kl}$ & $7.31 \mathrm{kl}$ & $3.64 \mathrm{kl}$ & $\begin{array}{c}3.84 \\
\text { no }\end{array}$ & $\begin{array}{c}16.98 \\
\mathrm{mn}\end{array}$ & $\begin{array}{c}16.63 \\
\mathrm{mn}\end{array}$ & $1.463 \mathrm{kl}$ \\
\hline & Hybrid 102 & $4.16 \mathrm{p}$ & $4.34 \mathrm{p}$ & $\begin{array}{c}2.27 \\
\mathrm{pq}\end{array}$ & $\begin{array}{c}2.36 \\
\mathrm{bc}\end{array}$ & $\begin{array}{c}16.26 \\
\text { no }\end{array}$ & $\begin{array}{c}15.94 \\
\text { no }\end{array}$ & $\begin{array}{c}1.210 \\
\operatorname{lm}\end{array}$ \\
\hline & Is 3214 & $4.04 \mathrm{p}$ & $4.26 \mathrm{p}$ & $1.96 \mathrm{q}$ & $2.05 \mathrm{u}$ & $14.38 \mathrm{p}$ & $13.93 \mathrm{p}$ & $\begin{array}{c}1.080 \\
\mathrm{mn}\end{array}$ \\
\hline \multirow[t]{4}{*}{ Azotobacter } & Giza2 & $6.30 \mathrm{mn}$ & $6.57 \mathrm{~lm}$ & $3.96 \mathrm{jk}$ & $\begin{array}{c}4.08 \\
\mathrm{mn}\end{array}$ & $\begin{array}{c}17.61 \\
\operatorname{lm}\end{array}$ & $17.22 \mathrm{kl}$ & $1.612 \mathrm{jk}$ \\
\hline & Piper & $7.15 \mathrm{jk}$ & $7.37 \mathrm{jk}$ & $4.36 \mathrm{ij}$ & $\begin{array}{c}4.40 \\
\operatorname{lm}\end{array}$ & $\begin{array}{c}20.09 \\
\mathrm{fg}\end{array}$ & $\begin{array}{c}19.75 \\
\text { fg }\end{array}$ & $1.798 \mathrm{hi}$ \\
\hline & Hybrid 102 & $5.21 \mathrm{o}$ & $5.45 \mathrm{o}$ & $\begin{array}{c}3.09 \\
\text { no }\end{array}$ & $\begin{array}{c}3.16 \\
\mathrm{qr}\end{array}$ & $18.87 \mathrm{ij}$ & $\begin{array}{c}18.99 \\
\mathrm{gh}\end{array}$ & $1.638 \mathrm{ij}$ \\
\hline & Is 3214 & $5.21 \mathrm{o}$ & $5.40 \mathrm{o}$ & $\begin{array}{c}2.98 \\
\text { op }\end{array}$ & $3.07 \mathrm{rs}$ & $17.93 \mathrm{kl}$ & $17.57 \mathrm{kl}$ & $1.460 \mathrm{kl}$ \\
\hline \multirow[t]{4}{*}{ Azospirillum } & Giza2 & $7.20 \mathrm{jk}$ & 7.38 jk & $4.42 \mathrm{ij}$ & $\begin{array}{c}4.49 \\
\mathrm{~lm}\end{array}$ & $\begin{array}{c}19.12 \\
\text { gh }\end{array}$ & $18.43 \mathrm{ij}$ & $\begin{array}{c}1.983 \\
\text { gh }\end{array}$ \\
\hline & Piper & $8.21 \mathrm{gh}$ & $8.37 \mathrm{hi}$ & $\begin{array}{c}4.92 \\
\text { gh }\end{array}$ & $5.04 \mathrm{ij}$ & 20.35 ef & $19.99 \mathrm{ef}$ & $2.392 \mathrm{~d}$ \\
\hline & Hybrid 102 & $6.36 \mathrm{~lm}$ & $6.58 \mathrm{~lm}$ & $\begin{array}{c}3.46 \\
\operatorname{lm}\end{array}$ & $\begin{array}{c}3.56 \\
\text { op }\end{array}$ & $\begin{array}{c}19.88 \\
\mathrm{fg}\end{array}$ & $\begin{array}{c}19.55 \\
\mathrm{fg}\end{array}$ & $\begin{array}{c}1.999 \\
\mathrm{fg}\end{array}$ \\
\hline & Is 3214 & $6.23 n$ & $6.43 \mathrm{mn}$ & $\begin{array}{c}3.26 \\
\text { no }\end{array}$ & $\begin{array}{c}3.37 \\
\mathrm{qr}\end{array}$ & $\begin{array}{c}19.07 \\
\text { gh }\end{array}$ & $18.65 \mathrm{hi}$ & $1.808 \mathrm{hi}$ \\
\hline \multirow[t]{3}{*}{ Mixture } & Giza2 & 8.08 hi & $8.29 \mathrm{hi}$ & $\begin{array}{c}5.59 \\
\text { de }\end{array}$ & $5.69 \mathrm{ef}$ & $\begin{array}{c}20.07 \\
\mathrm{fg}\end{array}$ & $\begin{array}{c}19.71 \\
\mathrm{fg}\end{array}$ & $\begin{array}{c}2.327 \\
\text { de }\end{array}$ \\
\hline & Piper & 9.17 ef & $9.37 \mathrm{fg}$ & $\begin{array}{c}6.33 \\
b c\end{array}$ & $\begin{array}{c}6.46 \\
b c\end{array}$ & $\begin{array}{c}21.27 \\
\text { de }\end{array}$ & $\begin{array}{c}20.97 \\
\text { de }\end{array}$ & $3.045 \mathrm{~b}$ \\
\hline & Hybrid 102 & $7.21 \mathrm{jk}$ & $7.45 \mathrm{ij}$ & $4.46 \mathrm{ij}$ & $4.57 \mathrm{kl}$ & $\begin{array}{c}20.80 \\
\text { de }\end{array}$ & $\begin{array}{c}20.43 \\
\text { de }\end{array}$ & $\begin{array}{c}2.347 \\
\text { de }\end{array}$ \\
\hline
\end{tabular}

Arab Univ. J. Agric. Sci., 13(3), 2005 
Abdel-Rahman; El-Shouny; Ashoub; Abdel-Gawad and Abd El-Maaboud

\begin{tabular}{|llllllllll|} 
Is 3214 & $7.12 \mathrm{jk}$ & $7.33 \mathrm{kl}$ & $4.09 \mathrm{jk}$ & $\begin{array}{c}4.16 \\
\mathrm{mn}\end{array}$ & $\begin{array}{c}20.04 \\
\mathrm{fg}\end{array}$ & $\begin{array}{c}\text { fg } \\
\text { fg }\end{array}$ & $2.124 \mathrm{ef}$ \\
\hline
\end{tabular}

Table 5. Cont.

\begin{tabular}{|c|c|c|c|c|c|c|c|c|}
\hline \multirow{22}{*}{$\begin{array}{c}\text { Salinity } \\
\text { of water } \\
\text { irrigation } \\
3700 \\
\text { ppm }\end{array}$} & \multirow[t]{2}{*}{$\mathrm{N}$} & \multirow[t]{2}{*}{ Varieties } & \multicolumn{2}{|c|}{ Fiber in stems $(\%)$} & \multicolumn{2}{|c|}{ Ash in leaves $(\%)$} & \multicolumn{2}{|c|}{ Ash in stems (\%) } \\
\hline & & & $1^{\text {st }}$ cut & $2^{\text {nd }}$ cut & $1^{\text {st }}$ cut & $2^{\text {nd }}$ cut & $1^{\text {st }}$ cut & $2^{\text {nd }}$ cut \\
\hline & \multirow[t]{4}{*}{$\begin{array}{l}\text { Recommend } \\
60 \mathrm{~kg} \mathrm{~N} / \text { fed. }\end{array}$} & Giza2 & $27.98 \mathrm{de}$ & $27.62 \mathrm{bc}$ & $\begin{array}{c}10.81 \\
\text { bc }\end{array}$ & $\begin{array}{c}11.37 \\
b c\end{array}$ & $13.85 \mathrm{bc}$ & $\begin{array}{c}12.65 \\
b\end{array}$ \\
\hline & & Piper & $30.96 \mathrm{a}$ & $30.42 \mathrm{a}$ & $12.32 \mathrm{a}$ & $12.78 \mathrm{a}$ & $15.27 \mathrm{a}$ & $14.09 \mathrm{a}$ \\
\hline & & Hybrid 102 & $29.17 \mathrm{ab}$ & $28.43 \mathrm{ab}$ & $\begin{array}{c}10.08 \\
\mathrm{~cd}\end{array}$ & $\begin{array}{c}10.55 \\
\text { de }\end{array}$ & $13.05 \mathrm{~d}$ & $\begin{array}{c}11.64 \\
\mathrm{~cd}\end{array}$ \\
\hline & & Is 3214 & 27.72 ef & $27.34 \mathrm{~cd}$ & $8.45 \mathrm{f}$ & $8.85 \mathrm{f}$ & $11.35 \mathrm{fg}$ & 10.32 \\
\hline & \multirow[t]{4}{*}{$\begin{array}{c}\text { Control } \\
\text { ( zero N) }\end{array}$} & Giza2 & $18.82 \mathrm{q}$ & $18.49 \mathrm{p}$ & $7.33 \mathrm{~g}$ & $7.72 \mathrm{hi}$ & $9.74 \mathrm{ij}$ & $8.42 \mathrm{ij}$ \\
\hline & & Piper & 21.44 no & $21.04 \mathrm{mn}$ & $8.13 \mathrm{fg}$ & $8.57 \mathrm{fg}$ & $10.80 \mathrm{gh}$ & $9.58 \mathrm{fg}$ \\
\hline & & Hybrid 102 & $18.88 \mathrm{q}$ & $18.42 \mathrm{p}$ & $5.95 \mathrm{kl}$ & $6.42 \mathrm{~lm}$ & $8.87 \mathrm{kl}$ & $7.37 \mathrm{kl}$ \\
\hline & & Is 3214 & $19.45 \mathrm{pq}$ & 18.85 op & $4.50 \mathrm{r}$ & $4.96 \mathrm{rs}$ & 7.47 no & $\begin{array}{c}6.24 \\
\text { no }\end{array}$ \\
\hline & \multirow[t]{4}{*}{ Azotobacter } & Giza2 & $23.89 \mathrm{kl}$ & $23.41 \mathrm{jk}$ & $8.43 \mathrm{f}$ & $8.83 \mathrm{f}$ & $11.32 \mathrm{fg}$ & $9.92 \mathrm{fg}$ \\
\hline & & Piper & $26.28 \mathrm{gh}$ & $25.83 \mathrm{ef}$ & $9.60 \mathrm{de}$ & $\begin{array}{c}10.20 \\
\text { de }\end{array}$ & $12.70 \mathrm{de}$ & $\begin{array}{c}11.15 \\
\text { de }\end{array}$ \\
\hline & & Hybrid 102 & $\begin{array}{c}22.73 \\
\mathrm{mn}\end{array}$ & $22.25 \mathrm{~lm}$ & $7.29 \mathrm{gh}$ & $7.81 \mathrm{gh}$ & $10.32 \mathrm{hi}$ & $\begin{array}{c}8.95 \\
\text { gh }\end{array}$ \\
\hline & & Is 3214 & $\begin{array}{c}22.74 \\
\mathrm{mn}\end{array}$ & $22.35 \mathrm{kl}$ & $6.04 \mathrm{kl}$ & $6.51 \mathrm{~lm}$ & $9.00 \mathrm{jk}$ & $7.95 \mathrm{jk}$ \\
\hline & \multirow[t]{4}{*}{ Azospirillum } & Giza2 & $25.84 \mathrm{hi}$ & $25.40 \mathrm{fg}$ & $9.45 \mathrm{e}$ & $9.92 \mathrm{e}$ & $11.91 \mathrm{ef}$ & $\begin{array}{c}10.43 \\
\text { ef }\end{array}$ \\
\hline & & Piper & $28.52 \mathrm{~cd}$ & $28.04 \mathrm{bc}$ & $\begin{array}{c}10.07 \\
\mathrm{~cd}\end{array}$ & $\begin{array}{c}10.55 \\
\text { de }\end{array}$ & $13.01 \mathrm{~d}$ & $\begin{array}{c}11.61 \\
\mathrm{~cd}\end{array}$ \\
\hline & & Hybrid 102 & $26.35 \mathrm{gh}$ & $25.97 \mathrm{ef}$ & $8.13 \mathrm{fg}$ & $8.61 \mathrm{fg}$ & $11.12 \mathrm{fg}$ & $9.62 \mathrm{fg}$ \\
\hline & & Is 3214 & $25.29 \mathrm{ij}$ & $24.89 \mathrm{hi}$ & $6.96 \mathrm{ij}$ & $7.44 \mathrm{ij}$ & $9.94 \mathrm{i}$ & $8.63 \mathrm{hi}$ \\
\hline & \multirow[t]{4}{*}{ Mixture } & Giza2 & $27.69 \mathrm{ef}$ & $27.15 \mathrm{~cd}$ & $\begin{array}{c}10.42 \\
\mathrm{~cd}\end{array}$ & $\begin{array}{c}10.83 \\
\mathrm{~cd}\end{array}$ & $13.22 \mathrm{~cd}$ & $\begin{array}{c}11.81 \\
\mathrm{bc}\end{array}$ \\
\hline & & Piper & $30.65 \mathrm{ab}$ & $30.25 \mathrm{a}$ & $11.33 \mathrm{~b}$ & $11.79 \mathrm{~b}$ & $14.17 \mathrm{~b}$ & $\begin{array}{c}12.47 \\
b c\end{array}$ \\
\hline & & Hybrid 102 & $29.41 \mathrm{ab}$ & $28.48 \mathrm{ab}$ & $9.61 \mathrm{de}$ & $\begin{array}{c}10.07 \\
\text { de }\end{array}$ & $12.55 \mathrm{de}$ & $\begin{array}{c}11.20 \\
\mathrm{de}\end{array}$ \\
\hline & & Is 3214 & $26.69 \mathrm{fg}$ & $26.20 \mathrm{de}$ & $8.08 \mathrm{fg}$ & $8.53 \mathrm{fg}$ & $11.03 \mathrm{gh}$ & $9.68 \mathrm{fg}$ \\
\hline
\end{tabular}

Arab Univ. J. Agric. Sci., 13(3), 2005 


\section{Sudan grass yield at Ras Sudr}

\begin{tabular}{|c|c|c|c|c|c|c|c|c|}
\hline \multirow{21}{*}{$\begin{array}{c}\text { Salinity } \\
\text { of water } \\
\text { irrigation } \\
9200 \\
\text { ppm }\end{array}$} & \multirow[t]{5}{*}{$\begin{array}{l}\text { Recommend } \\
60 \mathrm{~kg} \mathrm{~N} / \mathrm{fed} \text {. }\end{array}$} & Giza2 & $26.10 \mathrm{gh}$ & $25.51 \mathrm{fg}$ & $7.21 \mathrm{hi}$ & $7.67 \mathrm{ij}$ & $8.91 \mathrm{kl}$ & $7.63 \mathrm{jk}$ \\
\hline & & & & & & & & \\
\hline & & Piper & $30.10 \mathrm{ab}$ & $29.65 \mathrm{ab}$ & $7.54 \mathrm{gh}$ & $8.03 \mathrm{fg}$ & $9.04 \mathrm{jk}$ & $8.07 \mathrm{ij}$ \\
\hline & & Hybrid 102 & $29.99 \mathrm{ab}$ & $29.22 \mathrm{ab}$ & $6.78 \mathrm{ij}$ & $7.26 \mathrm{ij}$ & $8.27 \mathrm{kl}$ & $7.67 \mathrm{jk}$ \\
\hline & & Is 3214 & $29.38 \mathrm{ab}$ & $28.03 \mathrm{bc}$ & $6.69 \mathrm{ij}$ & $7.14 \mathrm{ij}$ & $8.13 \mathrm{~lm}$ & $7.19 \mathrm{kl}$ \\
\hline & \multirow[t]{4}{*}{$\begin{array}{l}\text { Control } \\
\text { ( zero N) }\end{array}$} & Giza2 & $17.78 \mathrm{q}$ & $17.21 \mathrm{p}$ & $3.46 \mathrm{u}$ & $3.90 \mathrm{u}$ & $4.91 \mathrm{v}$ & $4.02 \mathrm{t}$ \\
\hline & & Piper & $18.67 \mathrm{hi}$ & $18.15 \mathrm{p}$ & $4.03 \mathrm{tu}$ & $4.44 \mathrm{tu}$ & $5.49 \mathrm{uv}$ & $4.55 \mathrm{st}$ \\
\hline & & Hybrid 102 & $17.97 \mathrm{q}$ & $17.40 \mathrm{p}$ & $5.02 \mathrm{pq}$ & $5.46 \mathrm{pq}$ & $6.44 \mathrm{rs}$ & $\begin{array}{c}5.44 \\
\mathrm{pq}\end{array}$ \\
\hline & & Is 3214 & $17.88 \mathrm{q}$ & $17.18 \mathrm{p}$ & $4.42 \mathrm{st}$ & $4.84 \mathrm{st}$ & $5.81 \mathrm{tu}$ & $4.90 \mathrm{rs}$ \\
\hline & \multirow[t]{4}{*}{ Azotobacter } & Giza2 & $21.12 \mathrm{op}$ & 20.51 no & $5.07 \mathrm{op}$ & $5.48 \mathrm{pq}$ & $6.48 \mathrm{rs}$ & $5.25 \mathrm{qr}$ \\
\hline & & Piper & $25.57 \mathrm{ij}$ & $25.05 \mathrm{hi}$ & 5.35 no & $5.85 \mathrm{op}$ & $6.88 \mathrm{qr}$ & $\begin{array}{c}5.65 \\
\mathrm{pq}\end{array}$ \\
\hline & & Hybrid 102 & $24.76 \mathrm{ij}$ & $24.27 \mathrm{hi}$ & $5.21 \mathrm{no}$ & $5.71 \mathrm{op}$ & $6.67 \mathrm{qr}$ & $\begin{array}{c}5.69 \\
\mathrm{pq}\end{array}$ \\
\hline & & Is 3214 & $23.23 \mathrm{~lm}$ & $22.54 \mathrm{kl}$ & $4.77 \mathrm{qr}$ & $5.21 \mathrm{qr}$ & $6.21 \mathrm{st}$ & $5.26 \mathrm{qr}$ \\
\hline & \multirow[t]{4}{*}{ Azospirillum } & Giza2 & $24.31 \mathrm{jk}$ & $23.82 \mathrm{ij}$ & $5.88 \mathrm{~lm}$ & $\begin{array}{c}6.24 \\
\mathrm{mn}\end{array}$ & 7.26 po & $\begin{array}{c}6.13 \\
\text { op }\end{array}$ \\
\hline & & Piper & $27.71 \mathrm{ef}$ & $27.20 \mathrm{~cd}$ & 5.52 no & 6.04 no & $7.07 \mathrm{pq}$ & $\begin{array}{c}6.07 \\
\text { op }\end{array}$ \\
\hline & & Hybrid 102 & $28.32 \mathrm{~cd}$ & $27.82 \mathrm{bc}$ & $5.96 \mathrm{kl}$ & $6.38 \mathrm{~lm}$ & 7.39 op & $\begin{array}{c}6.47 \\
\mathrm{mn}\end{array}$ \\
\hline & & Is 3214 & $26.01 \mathrm{gh}$ & $25.41 \mathrm{fg}$ & $\begin{array}{c}5.68 \\
\mathrm{mn}\end{array}$ & $\begin{array}{c}6.11 \\
\mathrm{mn}\end{array}$ & $7.14 \mathrm{pq}$ & $\begin{array}{c}6.18 \\
\text { op }\end{array}$ \\
\hline & \multirow[t]{4}{*}{ Mixture } & Giza2 & $25.76 \mathrm{q}$ & $25.27 \mathrm{gh}$ & $6.86 \mathrm{ij}$ & $7.45 \mathrm{jk}$ & $8.47 \mathrm{kl}$ & $7.25 \mathrm{kl}$ \\
\hline & & Piper & $29.81 \mathrm{ab}$ & $29.24 \mathrm{ab}$ & $7.06 \mathrm{ij}$ & $7.50 \mathrm{ij}$ & $8.52 \mathrm{kl}$ & $7.30 \mathrm{kl}$ \\
\hline & & Hybrid 102 & $29.66 \mathrm{ab}$ & $28.94 \mathrm{ab}$ & $6.44 \mathrm{jk}$ & $6.85 \mathrm{kl}$ & $7.83 \mathrm{mn}$ & $\begin{array}{c}6.83 \\
1 \mathrm{~m}\end{array}$ \\
\hline & & Is 3214 & $28.71 \mathrm{bc}$ & $28.18 \mathrm{bc}$ & $6.49 \mathrm{jk}$ & $6.94 \mathrm{jk}$ & $7.88 \mathrm{mn}$ & $7.23 \mathrm{kl}$ \\
\hline
\end{tabular}

Arab Univ. J. Agric. Sci., 13(3), 2005 
Arab Univ. J. Agric. Sci., Ain Shams Univ., Cairo, 13(3), 755-769, 2005

nitrogen form. Giza2 and Piper varieties fertilized with the recommended dose of mineral nitrogen gave the highest mean values of protein percentage in leaves and stems at the two cuts. Whereas, Piper and Hybrid 102 varieties with mixture of biofertilizers had the highest mean values of protein percentage in leaves and stems at the second cut. On the other hand Hybrid102 and Is3214 varieties had the lowest mean values of protein percentage in leaves and stems under no nitrogen fertilization. Concerning fiber percentages, Piper variety gave the highest mean values of fiber percentages in leaves followed by Giza2 after treatment with the recommended dose of mineral nitrogen. Whereas, Piper and Hybrid 102 with the mixture of biofertilizers recorded the highest mean values of fibers percentages in leaves under salinity of irrigation water $(3700$ $\mathrm{ppm}$ ). On the other hand, Is3214 variety had the lowest mean values under the treatment without nitrogen fertilization at salinity of irrigation water $(9200 \mathrm{ppm})$. It is clear from results presented in Table (5) that Piper and Hybrid102 varieties gave the highest mean values of fibers percentages in stems with adding the recommended dose of mineral nitrogen followed by the mixture of biofertilizers treatment under salinity of irrigation water (3700ppm). While Giza2 and Is3214 varieties gave the lowest mean values of fiber percentages in stems under no nitrogen fertilization under saline water (9200 ppm). Similar results were obtained by Panwar et al (1999); Parasuraman et al (2000) and Patidar \& Mali (2004).

Data in Table (5) show that Piper variety gave the highest mean values of ash percentages in leaves and stems followed by Giza2 on the recommended dose of mineral nitrogen fertilizer and Piper on the mixture of biofertilizers under saline water $3700 \mathrm{ppm}$ in the both cuts. Whereas, Giza2 had the lowest mean values of ash percentages in leaves and stems followed by Piper under treatment without nitrogen fertilization of irrigation water 9200ppm. Patel et al (1975); Patel \& Rajagopal (2003) and Ramesh \& Sammi (2004) demonstrated that chemical composition of leaves in addition to familiar dilution effects and ion competition revealed increased accumulation of $\mathrm{Ca}, \mathrm{Na}$, and $\mathrm{Cl}$ related to high level of $\mathrm{P}$ supply at high salinity conditions only.

\section{REFERENCES}

A.O.A.C., Association of Official
Analytical Chemists, (1990). Methods
of Analysis, $15^{\text {th }}$ Ed., pp. 1045-1106.
Washington D.C.,USA.

Abbas, M.T.; A. Rammah; M. Monib;

E.H. Ghanem; M.A.M. Eid; M.F.Z.

Emara and N.A. Hegazi (1993). International Symposium N2-Fixation, pp. 485-487. Cairo. Univ. Press, Egypt. Barik, A.K.; A.K. Mukherjee and B.K. Mandal (1998). Growth and yield of

1- Desert Research Center, Matariya, Cairo, Egypt

2- Department of Agronomy, Faculty of Agriculture, Ain Shams University, Shoubra El-Kheima, Cairo, Egypt. 
sorghum (Sorghum bicolor) and groundnut (Arachis hypogaea) grown as sole and intercrops under different nitrogen regimes. Indian J. of Agron., 43(1): 27-32.

Bates, L.S.; R.P. Waldren and I.D.

Teare (1973). Rapid determination of free proline for water stress studies.

Plant and Soil, 39: 205-207.

Datta, K.S.; A. Kumar; S.K. Varma and R. Angrish (1996). Studies on salt tolerance of three tropical forage crops on the basis of mineral ion uptake. Forage Res., 22 (2\&3): 129-138.

Dahiya, O.S.; B.S. Dahiya; R.C. Punia and C. Ram (1997). Respiration rate as a measure of seedling establishment in forage sorghum (Sorghum bicolor (1.) Moench). Forage Res.,23(3\&4):149-152. Desale, J.S.; R.M. Babar; S.H. Pathan and R.L. Bhilare (1999). Effect of biofertilizers with various nitrogen levels on green forage yield of sorghum. Forage Res., 24 (4): 195-198.

Duncan, D.B. (1955). Multiple range and multiple "F" tests. Biometrics, pp. 1-42.

Francois, L.E.; T. Donovan and E.V. Maas. (1984). Salinity effects on seed yield, growth and germination of grain sorghum. Agron. J., 76: 742-754.

Hassan, S.A. (1994). Studies on Some Sorghum Mutation Types Induced by Gamma-ray and Ethylamine Treatments under Stress Conditions. pp. 22-23 \& 53-59. Ph.D. Thesis Moshtohor, Egypt.

Kaoud, E.E. and M.A.B. El-Fieshawy (1990). Influence of soil salinity on growth and mineral composition of sorghum (sorghum bicolor, L.). Egypt. J. Appi. Sci., 5 (2): 256-271.

Karwasra, R.S. and D.R. Dahiya (1997). Performance of forage sorghum (Sorghum bicolor) varieties under nitrogen fertilization. Forage Res., 23 (1\&2): 121-123.

Karwasra, R.S.; R.S. Kadian and D.R. Dahiya (1996). Effect of varieties and nitrogen on yield and yield attributes in forage sorghum. Forage Res., 22 (2\&3): 203-206.

Kramer, P.J. (1969). Plant and Soil Water Relationships: A Modern Synthesis. pp. 102-168. McGraw-Hill Book, Co., Inc., New York.

Kumar, A.; K.S. Datta; J. Gorham; R.G. Wyn Jones and P.A. Hollington (1991): Influence of salinity levels at two growing stages on the performance of three tropical forge crops. J. Indian Soc. Coastal Agric. Res., 9: 493-502. Mustafa, S.M.I. and N. Shaheen (1984). Physiological and physiochemical evaluations of four sorghum cultivars. $\boldsymbol{J}$. Agric. Res. Tanta Univ., 10 (1): 1-11.

Nassar, Z.M.; M.N. Nour El-Dein and A.A. El-Houssini (2000). Effect of moisture stress and presoaking with IAA on forage yield of Sorghum bicolor (L.) Moench grown in salt affected soils at Wadi-Sudr. Annals Agric. Sci., Ain Shams Univ., Cairo, 45 (1): 201-214. Panwar, V.S.; Tewatin, B.S. and Lodhi, G.P. (1999). Chemical composition and in vitro dry matter digestibility of some varieties of Sorghum fodder harvested at different stages. Forage Res., 24(4): 209211.

Parasuraman, P.; P. Duraisamy and A.K. Mani (2000). Effect of organic, inorganic and bio-fertilizers on soil fertility under double-cropping system in rainfed red soils. Indian J. of Agron., 45 (2): 242-247.

Patel, P.M.; A. Wallace and E.F. Wallihan (1975). Influence of salinity and N-P fertility levels on mineral 
Abdel-Rahman; El-Shouny; Ashoub; Abdel-Gawad and Abd El-Maaboud

content and growth of sorghum in sand culture. Agron. J., 67: 622-625.

Patel, P.C.; J.R. Patel and A.C. Sadhu (1992). Response of forage sorghum (Sorghum bicolor) to biofertilizer and nitrogen levels. Indian J. Agron., 37 (3): 466-469.

Patel, J.R. and S.J. Rajagopal (2003).

Nitrogen management for production of sorghum (Sorghum bicolor) and cowpea (Vigna unguiculata) forage under intercropping system. Indian J. Agron., 48 (1): 43-37.

Patidar, M. and A.L. Mali (2004).

Effect of farmyard manure, fertility levels and boi-fertilizers on growth, yield and quality of sorghum (Sorghum bicolor).

Indian J. Agron., 49 (2): 117-120.

Peach, K. and M. V. Tracey (1956).

Modern Methods of Plant Analysis. Vol. 1, pp. 13-19, Springer Verlag Berlin.

Ramamurthy, V. (2002). Effect of nitrogen and Azospirillum inoculation on growth and green forage yield of Pennisetum trispecific hybrid. Indian $\mathbf{J}$. Agron., 47 (4): 566-570.

Ram, S.N. and Bhagwan Singh (2003). Physiological growth parameters, forage yield and nitrogen uptake of sorghum (Sorghum bicolor) as influenced with legume intercropping, harvesting time and nitrogen level. Indian J. Agron., 48 (1): 38-41.

Ramesh, P. and K.R. Sammi (2004).

Productivity and nutrient-use efficiency of soybean (Glycin max) and sorghum (Sorghum bicolor) intercropping at different levels of nitrogen in rainfed deep vertisols. Indian J. Agron., 49 (1): 31-33.

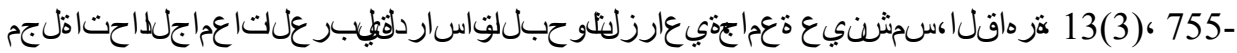
769، 2005
Rawat, C.R. and C.R. Hazra (1998). Effect of nitrogen and Azotobacter on forage yield of oats. Forage Res., 23 (3\&4): 241-243. Saffa, M. Ismaeil; Om Mohamed A. Khafagi and Samya M. Sohsah (1993). Effect of some seed hardening treatments on germination, growth and yield of Sudan grass grown under saline conditions. Desert Inst. Bull., 43 (2): 221-242.

Smith, D.; G. M. Paulsen and C. A. Roguse (1964). Extraction of total available carbohydrates from grass and legume tissues. Plant Physiol., 39: 960962.

Steel, R. G. D. and J. H. Torrie (1980). Principles and Procedures of Statistics, $2^{\text {nd }}$ Ed. 633 pp., McGraw-Hill Book Co., Inc., New York.

Subba Rao, N.S.; K.V.R. Tilak and M.L. Kumari (1979). Effect of biofertilizers with various nitrogen levels on root growth and forage yield of sorghum. Curr. Sci., 48: 133-134. 
Arab Univ. J. Agric. Sci., Ain Shams Univ., Cairo, 13(3), 755-769, 2005

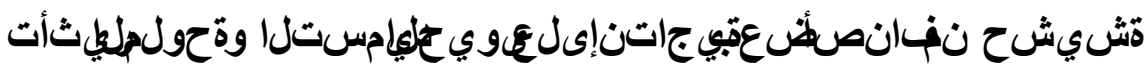

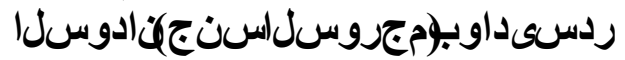

]50[

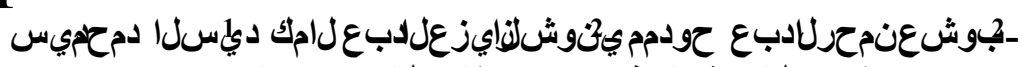

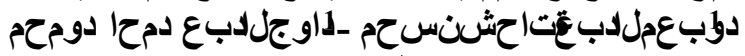

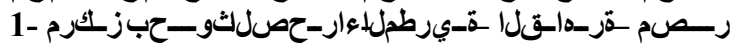

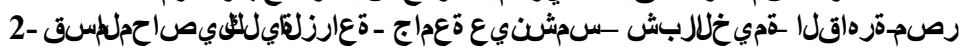

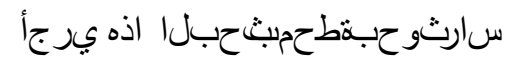

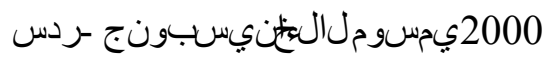

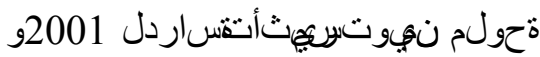

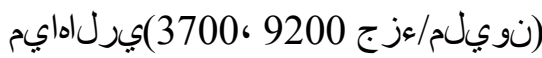

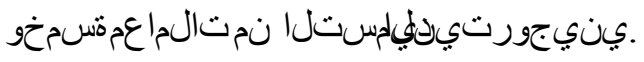

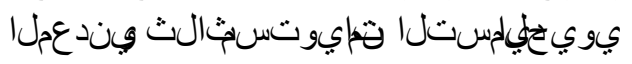

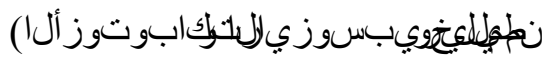

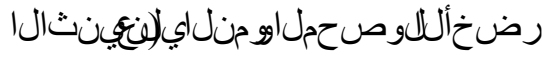

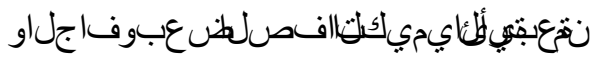

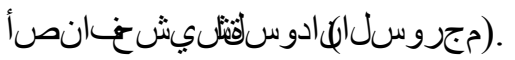

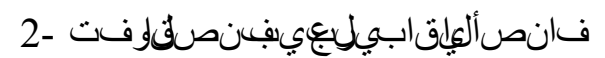

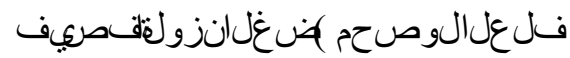

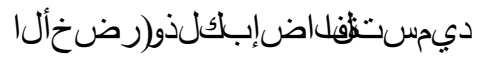

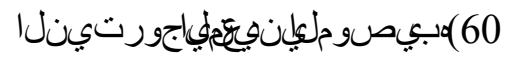

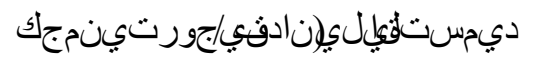

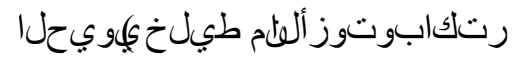

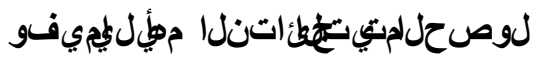 \\ اهيل

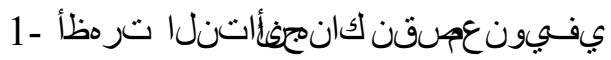

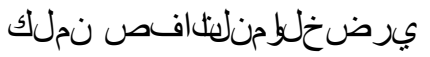

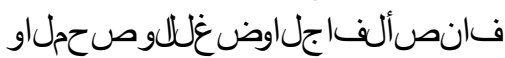

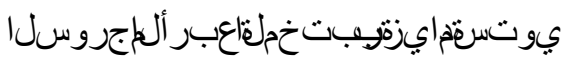 \\ 9200بيإ 3700 نمبيرل|هابيمةتحولم

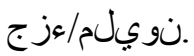

1- Desert Research Center, Matariya, Cairo, Egypt

2- Department of Agronomy, Faculty of Agriculture, Ain Shams University, Shoubra El-Kheima, Cairo, Egypt.

(Received May 25, 2005)

(Accepted June 6, 2005) 
Arab Univ. J. Agric. Sci., Ain Shams Univ., Cairo, 13(3), 755-769, 2005

$$
\begin{aligned}
& \text { ي يلنألبياتش حللجلنيع (بيبسوزيال } \\
& \text { هايمة حولجيوتست حتك تلتجيوناثل } \\
& \text { نويلم /ءزج 9200و 3700يرلا. }
\end{aligned}
$$

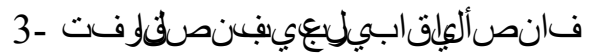

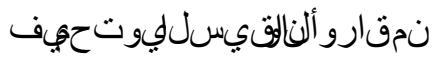

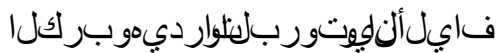

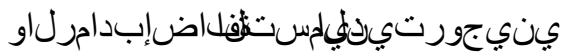

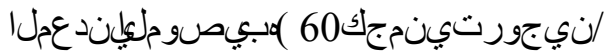

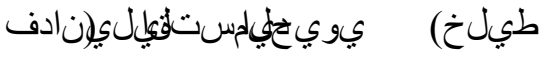

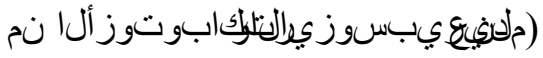

لكت حتكلثنيناثل اويلنونألياتش حل
9200و 3700ةحوليجهوتسم نم

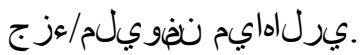

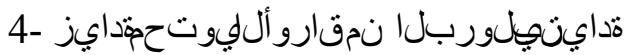

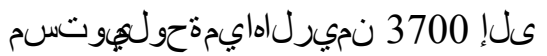

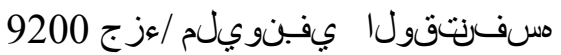

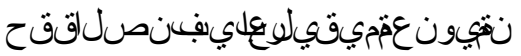

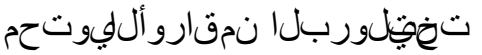

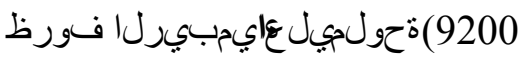

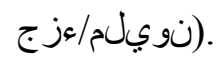

\section{ىفطصأهي عامسإ دمحبيل ع نزمجيك حت \\ ملادبعززوف دمحم رهظم د.}

1- Desert Research Center, Matariya, Cairo, Egypt

2- Department of Agronomy, Faculty of Agriculture, Ain Shams University, Shoubra El-Kheima, Cairo, Egypt. 